\section{Mesolimbic dopamine reward system hypersensitivity in individuals with psychopathic traits}

Joshua W Buckholtz ${ }^{1,2}$, Michael T Treadway ${ }^{1}$, Ronald L Cowan ${ }^{1,3}$, Neil D Woodward ${ }^{3}$, Stephen D Benning ${ }^{1}$, Rui Li ${ }^{4}$, M Sib Ansari ${ }^{4}$, Ronald M Baldwin ${ }^{4}$, Ashley N Schwartzman ${ }^{1}$, Evan S Shelby ${ }^{1}$, Clarence E Smith ${ }^{4}$, David Cole ${ }^{5}$, Robert M Kessler ${ }^{4}$ \& David H Zald ${ }^{1,3}$

Psychopathy is a personality disorder that is strongly linked to criminal behavior. Using $\left.{ }^{18} \mathrm{~F}\right]$ fallypride positron emission tomography and blood oxygen level-dependent functional magnetic resonance imaging, we found that impulsiveantisocial psychopathic traits selectively predicted nucleus accumbens dopamine release and reward anticipationrelated neural activity in response to pharmacological and monetary reinforcers, respectively. These findings suggest that neurochemical and neurophysiological hyper-reactivity of the dopaminergic reward system may comprise a neural substrate for impulsive-antisocial behavior and substance abuse in psychopathy.

The net annual burden of crime in the US has been estimated to exceed $\$ 1$ trillion $^{1}$, making criminal behavior a costly large-scale social problem and a critical target for scientific investigation. Although the risk architecture underlying criminality is complex, psychopathy has emerged as a particularly robust predictor of criminal behavior and recidivism. Psychopathy is a personality disorder characterized by a combination of superficial charm, persistent instrumental antisocial behavior, marked sensation-seeking and poor reflection, blunted empathy and punishment sensitivity, and shallow emotional experiences ${ }^{2}$. Recent research on the neural substrates of psychopathy has focused on the profound emotional deficits seen in psychopaths and has emphasized the possible contributions of amygdala and ventromedial prefrontal cortex dysfunction to deficient fear processing and empathy ${ }^{3}$. However, although emotional and interpersonal deficits are often considered to be core features of the disorder, the empirical linkage of such deficits to criminality (particularly, to risk for committing violent crimes) is mixed $^{4-6}$. In contrast, the impulsive-antisocial facets of the disorder more consistently predict many of the most socially problematic behaviors associated with psychopathy, including violent criminal offending $^{5-7}$. To date, the neural underpinnings of this impulsiveantisocial dimension remain essentially unknown.
Prior research has also shown that psychopathic individuals have a markedly increased risk of developing substance use problems ${ }^{8}$. Such associations mirror preclinical work demonstrating that impulsive traits predict enhanced susceptibility to drug-seeking and relapse ${ }^{9}$. Given the strong link between psychopathy and substance abuse, previous studies indicating that the mesolimbic dopamine (DA) system is important in the pathophysiology of substance use disorders and evidence that individual differences in the mesolimbic DA system predispose the development of substance abuse ${ }^{9}$, we hypothesized that psychopathic traits would be associated with dysfunction in mesolimbic DA reward circuitry. To test the prediction that individuals with psychopathic traits are characterized by alterations in mesolimbic DA neurochemistry and neurophysiology, we used positron emission tomography (PET) imaging of psychostimulantinduced DA release, in concert with a functional magnetic resonance imaging (fMRI) probe of the reward system. Psychopathic traits were measured with the psychopathic personality inventory (PPI), a wellvalidated trait measure of psychopathy, in a sample of community volunteers with no prior history of substance abuse (see Supplementary Data and Supplementary Discussion). Prior studies have shown that the PPI is composed of two underlying latent factors: a 'fearless dominance' (PPI-FD) factor indexing emotional-interpersonal facets of psychopathy and an 'impulsive antisociality' (PPI-IA) factor linked to socially deviant behavior ${ }^{10}$. Furthermore, such work has shown that the PPI-IA (but not the PPI-FD) factor is selectively associated with aggression, impulsivity and substance abuse in both incarcerated and community samples ${ }^{7,10}$. Given that animal studies have linked impulsiveaggressive traits to substance abuse and nucleus accumbens (NAcc) DA function ${ }^{9,11}$, we hypothesized that PPI-IA factor scores would selectively predict amphetamine-induced DA release in the NAcc.

To examine the relationship between psychopathic traits and DA release, we performed voxel-wise correlation analyses between PPI factor scores and maps of the percentage change in $\left[{ }^{18} \mathrm{~F}\right]$ fallypride binding potential between placebo and amphetamine $(0.43 \mathrm{mg}$ per $\mathrm{kg}$ of body weight; two-day, single-blind protocol, $n=30$; Supplementary Methods and Supplementary Fig. 1) in an anatomical NAcc region of interest (ROI; Supplementary Fig. 2). PPI-IA scores were strongly correlated with amphetamine-induced DA release in bilateral NAcc (Fig. 1, see Supplementary Methods and Supplementary Data). However, no relationship was observed between participants' PPI-FD scores and amphetamine-induced NAcc DA release, even at a liberal statistical threshold of $P<0.1$ (uncorrected for multiple comparisons). Tests for differences in dependent correlations confirmed that the correlation between NAcc release and PPI-IA was significantly stronger than the correlation between NAcc DA release and PPI-FD (left NAcc, $t_{27}=3.87$; right NAcc, $t_{27}=2.86 ; P<0.05$; calculated using per-subject mean DA release values from entire anatomical NAcc ROI; Supplementary Fig. 3). Thus, trait psychopathy is associated with

${ }^{1}$ Department of Psychology, ${ }^{2}$ Vanderbilt Brain Institute, ${ }^{3}$ Department of Psychiatry, ${ }^{4}$ Department of Radiology and ${ }^{5}$ Department of Psychology and Human Development, Vanderbilt University, Nashville, Tennessee, USA. Correspondence should be addressed to J.W.B. (joshua.buckholtz@vanderbilt.edu). 
a

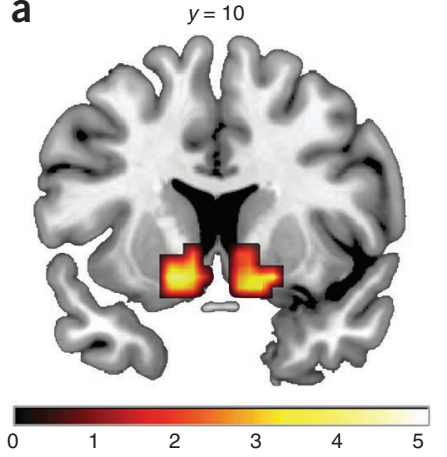

b

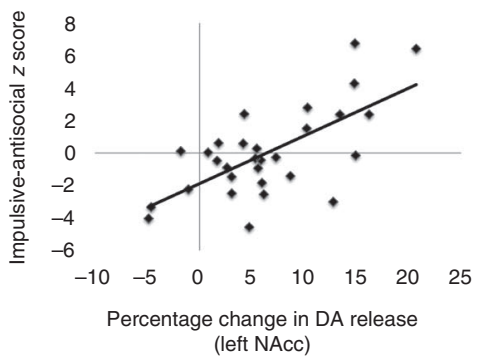

C

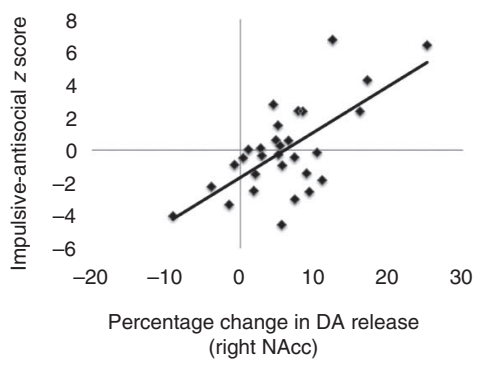

Figure 1 Impulsive-antisocial traits predict nucleus accumbens DA release. (a) Statistical parametric map (SPM) showing that PPI-IA factor Scores selectively predicted increased amphetamine-induced DA release in bilateral NAcc (left NAcc: $-16,10,-10, p_{\text {false discovery rate }}=0.003, z=3.74$, cluster size $(k)=56$; right NAcc: $16,18,-6$; $p_{\text {false discovery rate }}=0.002, z=4.21, k=44$ ). All coordinates reference the coordinate system of the Montreal Neurological Institute. SPM thresholded at $P<0.05$ (uncorrected) for visualization purposes. Color bar indicates $t$ statistic value. (b,c) Scatter plot depicting the relationship between PPI-IA factor scores and amphetamine-induced DA release in left (b) and right (c) NAcc. DA release values were extracted from clusters defined by a $p_{\mathrm{FDR}}<0.05$ threshold.

NAcc DA hyper-reactivity and this relationship is selective for the impulsive-antisocial dimension of this construct.

The observed trait-associated hypersensitivity to pharmacologically induced DA release in the NAcc raised the possibility that impulsiveantisocial traits might be linked to reward-related information processing biases in this region. Specifically, in light of recent findings of coupling between NAcc DA release and NAcc blood oxygen level-dependent (BOLD) signal ${ }^{12}$, we predicted that individuals with high levels of impulsive-antisocial traits would show enhanced NAcc recruitment in response to monetary reinforcement. To test this possibility, we scanned 24 subjects with fMRI during the monetary incentive delay task, which allows assessment of the extent of ventral striatal (NAcc) engagement by reward (Supplementary Methods). To examine the relationship between psychopathic traits and reward-related NAcc BOLD signal, we calculated the percent change in NAcc BOLD signal for each subject (mean percent change value in the anatomical ROI mentioned above) during reward anticipation trials (reward versus no reward contrast). Consistent with our PET data, we found a strong correlation between PPI-IA factor scores and BOLD signal in the right NAcc during reward anticipation $(r=0.63, P=0.001$; Fig. 2, Supplementary Figs. 4 and 5 and Supplementary Data). The correlation between left NAcc BOLD signal and PPI-IA was also nominally significant $(r=0.44, P=0.03)$, but did not survive correction for multiple correlation tests (Supplementary Methods). Further mirroring the PET data, we did not find any relationship between PPI-FD factor scores and NAcc BOLD signal during reward anticipation (right NAcc, $r=-0.02, P=0.94$; left NAcc, $r=-0.18, P=0.41$ ). A test for differences in dependent correlations revealed a significant difference in the correlations between right NAcc BOLD and PPI-IA versus PPI-FD scores $\left(t_{21}=2.68, P<0.05\right.$; Supplementary Fig. 6). Notably, we found no significant relationships between either PPI-IA or PPI-FD and BOLD signal during the reward feedback phase of the task in either the NAcc or in the medial prefrontal cortex (see Supplementary Data and Supplementary Fig. 7). These data indicate that NAcc information processing is selectively biased toward the anticipation of (that is, the motivated preparation to obtain) potential rewards in individuals with high levels of impulsive-antisocial, but not fearless dominance, traits.

The correspondence between trait correlations for the PET and fMRI measures suggested that NAcc DA release and BOLD signal might themselves be linked. There was both fMRI and PET data available for 18 individuals. For these participants, we examined the relationship between amphetamine-induced NAcc DA release and reward anticipation-related BOLD signal by extracting signal estimates from the NAcc ROI used above. Correlation analysis confirmed that there was a significant positive relationship between right NAcc DA release and right NAcc BOLD signal during reward anticipation $(r=0.49, P=0.02$; Supplementary Fig. 8), consistent with recent reports that NAcc engagement during reward anticipation may be at least partially driven by DA release ${ }^{12}$.

Taken together, our PET and fMRI data indicate that one specific factor in the larger psychopathy trait construct, impulsive antisociality, is associated with the neurochemistry and function of the NAcc. However, we were concerned that our findings might result from variation in related, but more general, personality constructs that do not necessarily access antisocial behavior or aggression, rather than from our principal construct of interest (the impulsive-antisocial dimension of psychopathy). We therefore used multiple-regression analyses to control for the potentially confounding effects of individual differences in attentional/cognitive impulsivity (measured using the Barratt Impulsiveness Scale), novelty-seeking (measured using the novelty-seeking scale from the Tridimensional Personality Questionnaire) and extraversion (measured using the extraversion scale
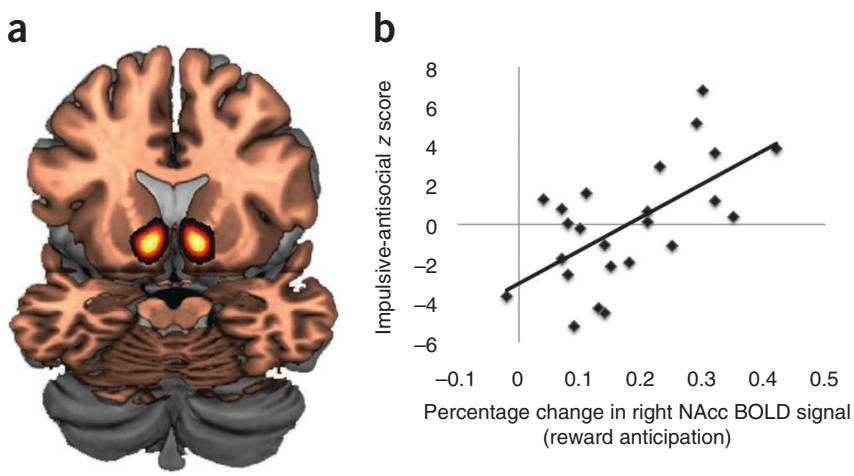

Figure 2 Impulsive-antisocial factor scores are selectively associated with NAcc BOLD signal during monetary reward anticipation. (a) Image depicts the Harvard-Oxford nucleus accumbens anatomical ROI from which BOLD signal estimates were obtained. (b) Scatter plot depicts the relationship between PPI-IA factor scores and reward anticipation-related BOLD signal in the right NAcc. 


\section{BRIEF COMMUNICATIONS}

from the NEO Personality Inventory Revised). PPI-IA remained a significant predictor of NAcc amphetamine-induced DA release and reward-related BOLD signal even after adjusting for these scores (all $P$ values $<0.05$; see Supplementary Data). On the whole, the pattern of these correlations suggests a unique link between impulsive-antisocial temperament and mesolimbic DA system hypersensitivity to pharmacological and monetary rewards that is at least partially independent of the effects of purely attentional or cognitive impulsivity and from the effects of other higher-order or cardinal personality traits that are conceptually or empirically related to DA.

Our data indicate that DA is an important neurochemical modulator of individual differences in human antisocial personality traits, with impulsive-antisocial temperament predicting excess neurochemical and functional engagement of the mesolimbic DA system in response to reward. Although a generation of clinical work has outlined a clear role for serotonin in impulsive violence, the role of DA in human antisociality has been largely overlooked. However, mounting preclinical research in rodents suggests that mesolimbic DA is critical for the expression of aggression: DA is released during aggressive episodes $^{13}$, NAcc DA blockade attenuates aggressive responding ${ }^{14}$, trait differences in aggression are associated with NAcc DA levels ${ }^{11}$ and genetic manipulations that reduce striatal DA clearance increase aggressive behavior ${ }^{15}$. Although aggression and antisociality are not synonymous, aggression is a strong behavioral correlate of impulsive-antisocial traits, as demonstrated by the fact that PPI-IA scores predict aggressive behavior in both incarcerated and community samples ${ }^{7}, 10$. Furthermore, insofar as we found that antisocial traits are tied to alterations in a brain system that is compromised in addiction, these results suggest a neurobiological mechanism that mediates the linkage between substance abuse and personality disorders characterized by antisocial behavior.

How might mesolimbic DA hyper-reactivity lead to the development of a psychopathic personality style? We propose a model that treats heightened stimulated NAcc DA release as a stable trait, which in turn leads to excessive recruitment of mesolimbic reward circuitry by behaviorally relevant environmental reinforcers (Supplementary Discussion). This hypersensitivity could be a result of either an intrinsic hyper-reactivity of midbrain DA neurons (that is, heightened phasic midbrain DA neuron burst firing to potentially rewarding stimuli) or diminished regulatory control of NAcc dopaminergic function stemming from a more widespread failure of inhibitory mechanisms (for example, dysregulation of inhibitory afferents to the ventral tegmental area). Whatever the proximal mechanism, magnified brain responses during reward anticipation might lead to enhanced motivation to obtain reward; this predisposition, in concert with reduced sensitivity in brain regions responsible for detecting the emotions of others and involved in learning from aversive outcomes ${ }^{3}$, could lead to the instrumental style of aggression that is common in psychopaths. By the same token, given evidence that aggression may itself have reinforcing properties that are similar in some ways to drugs of abuse ${ }^{14}$, neurochemical and neurophysiological hypersensitivity to reinforcers in the mesolimbic DA system may promote the development of persistent aggression in parallel with its putative influence on substance abuse susceptibility.

Note: Supplementary information is available on the Nature Neuroscience website.

\section{ACKNOWLEDGMENTS}

We thank B. Knutson for kindly making the monetary incentive delay task available and C. Weiner and M. McHugo for assistance with fMRI scanning and data analysis. This research was funded by the National Institute on Drug Abuse (R01DA019670-04).

\section{AUTHOR CONTRIBUTIONS}

J.W.B., R.M.K. and D.H.Z. designed the study. E.S.S. and A.N.S. recruited participants into the study and collected PET and personality data. J.W.B. collected fMRI data with assistance from E.S.S., and A.N.S., R.L., N.D.W. and R.M.K. performed single-subject PET data analysis and quality control. J.W.B. performed group level PET data analysis with assistance from M.T.T. J.W.B. analyzed fMRI data at all stages. M.S.A. and R.M.B. synthesized radiolabeled fallypride for PET scanning. S.D.B. provided conceptual advice, statistical support and supplementary analyses for the PPI data. R.L.C. oversaw all medical aspects of the protocol. C.E.S. and R.M.K. provided medical support for PET scanning. D.C. provided conceptual support and statistical advice for the study. J.W.B., M.T.T. and D.H.Z. wrote the manuscript with assistance from R.L.C.

\section{COMPETING FINANCIAL INTERESTS}

The authors declare no competing financial interests.

Published online at http://www.nature.com/natureneuroscience/.

Reprints and permissions information is available online at http://www.nature.com/ reprintsandpermissions/.

1. Anderson, D.A. J. Law Econ. 42, 611-642 (1999).

2. Hare, R.D. Psychiatr. Clin. North Am. 29, 709-724 (2006).

3. Blair, R.J. Phil. Trans. R. Soc. Lond. B Biol. Sci. 363, 2557-2565 (2008).

4. Neumann, C.S. \& Hare, R.D. J. Consult. Clin. Psychol. 76, 893-899 (2008).

5. Walters, G.D. Law Hum. Behav. 27, 541-558 (2003).

6. Skeem, J.L. \& Mulvey, E.P. J. Consult. Clin. Psychol. 69, 358-374 (2001).

7. Edens, J.F., Poythress, N.G. Jr., Lilienfeld, S.O. \& Patrick, C.J. Behav. Sci. Law 26, 529-541 (2008).

8. Smith, S.S. \& Newman, J.P. J. Abnorm. Psychol. 99, 430-439 (1990).

9. Everitt, B.J. et al. Phil. Trans. R. Soc. Lond. B 363, 3125-3135 (2008).

10. Benning, S.D., Patrick, C.J., Hicks, B.M., Blonigen, D.M. \& Krueger, R.F. Psychol. Assess. 15, 340-350 (2003).

11. Lewis, M.H., Gariepy, J.L., Gendreau, P., Nichols, D.E. \& Mailman, R.B. Neuropsychopharmacology 10, 115-122 (1994).

12. Schott, B.H. et al. J. Neurosci. 28, 14311-14319 (2008).

13. Ferrari, P.F., van Erp, A.M., Tornatzky, W. \& Miczek, K.A. Eur. J. Neurosci. 17 371-378 (2003)

14. Couppis, M.H. \& Kennedy, C.H. Psychopharmacology (Berl.) 197, 449-456 (2008).

15. Rodriguiz, R.M., Chu, R., Caron, M.G. \& Wetsel, W.C. Behav. Brain Res. 148, 185-198 (2004). 